\title{
Immune responses to a recombinant, four-component, meningococcal serogroup \\ 2 B vaccine (4CMenB) in adolescents: A phase III, randomized, multicentre, lot-to- 3 lot consistency study
}

Kirsten P Perrett MBBS, PhD, FRACP ${ }^{1}$, Jodie McVernon PhD FAFPHM ${ }^{1}$, Peter C Richmond MBBS, MRCP, FRACP ${ }^{2}$, Helen Marshall MBBS, MD, MPH, DCH ${ }^{3}$, Michael Nissen, BMedSc, MBBS, FRACP, FRCPA, FFSc ${ }^{4}$, Allison August MD ${ }^{5}$, Sandra Percell $\mathrm{PhD}^{5}$, Daniela Toneatto $\mathrm{MD}^{6}$, Terry Nolan MBBS, PhD, FRACP, FAFPHM $^{1}$

Affiliations: ${ }^{1}$ Vaccine and Immunisation Research Group (VIRGo), Murdoch Childrens Research Institute and Melbourne School of Population and Global Health, The University of Melbourne, Melbourne, Australia,

${ }^{2}$ School of Paediatrics and Child Health, University of Western Australia, Wesfarmers Centre of Vaccines and Infectious Diseases, Telethon Kids Institute, Princess Margaret Hospital for Children, Perth, Australia;

${ }^{3}$ Vaccinology and Immunology Research Trials Unit (VIRTU), Women's and Children's Hospital, School of Paediatrics and Reproductive Health and Robinson Research Institute, University of Adelaide, Adelaide, South Australia;

${ }^{4}$ Queensland Paediatric Infectious Diseases Laboratory (Qpid), Queensland Children's Medical Research Institute, Royal Children's Hospital, University of Queensland, Brisbane, Australia. ${ }^{5}$ Novartis Vaccines and Diagnostics Inc., Cambridge MA USA, ${ }^{6}$ Novartis Vaccines and Diagnostics S.r.l., Siena, Italy.

Address correspondence to: Professor Terry Nolan Head, Melbourne School of Population and Global Health, Level 5, 207 Bouverie Street, The University of Melbourne 3010, Victoria, Australia. +61 38344

Short title: MenB vaccine kinetics in adolescents

Abbreviations: MenB- serogroup B meningococcal, fHbp - factor $\mathrm{H}$ binding protein, NadA - Neisserial adhesin A, NHBA - Neisseria heparin binding antigen, PorA porin A, hSBA- serum bactericidal assay using human complement, GMC- geometric mean concentration, GMT-geometric mean titre

Keywords: Neisseria meningitidis, serogroup B meningococcal vaccine, immunity, immunization, adolescence 
44 Funding sources: This study was funded by Novartis Vaccines. Dr Perrett, and A/Professors McVernon and Marshall (1016272) are recipients of Research Fellowships from the Australian National Health and Medical Research Council.

\section{Potential conflict of interest:}

Dr Perrett has received honoraria from Pfizer for educational lectures. Dr Perrett, A/Professor McVernon and Professor Nolan's institution (MCRI) has received research grants from GSK, Novartis, CSL, Pfizer and Sanofi Pasteur.

A/Professor Richmond has received institutional funding for investigator-initiated research from GlaxoSmithKline Biologicals, Novartis, Pfizer and Merck, received

A/Professor McVernon has been an investigator on vaccine and epidemiological studies sponsored by a range of vaccine manufacturers, and in this role has received support for conference attendance, presentation of data and membership of vaccine advisory boards. A/Professor McVernon is a member of ATAGI.

A/Professor Marshall has been a member of vaccine advisory boards for GlaxoSmithKline and Novartis. A/Professor Marshall's institution (Women's and Children's Hospital) has received research grants from GlaxoSmithkline, Novartis, Pfizer and Sanofi Pasteur. A/Professor Helen Marshall has received travel support from Pfizer and Novartis for conference attendance and presentation of independent scientific data.

A/Professor Nissen directs the Queensland Paediatric Infectious Diseases laboratory that has performed the Meningococcal Antigen Testing System assay on Australian isolates causing invasive meningococcal disease on the behalf of Novartis. He has received travel support from GSK and Pfizer for conference attendance and presentation of data of independent research at international meetings, honoraria from bioCSL, Novartis and Pfizer for educational lectures, institutional funding for investigator-initiated research from Abbott Australasia, as well as been an principal investigator on vaccine and epidemiological studies sponsored by a range of vaccine manufacturers, and in this role has received support for conference attendance, presentation of data and membership of vaccine advisory boards. A/Professor Nissen is the current Chair of the Australian National Verification Committee for Measles Eradication and a past member of ATAGI.

Professor Nolan chaired (until June 2014) the Australian Government's Technical Advisory Group on Immunization (ATAGI) and is a member of the World Health Organization Strategic Advisory Group of Experts (SAGE) on Immunization.

Dr Allison August is a former employee of the study sponsor. Dr Daniela Toneatto is an employee of Novartis Vaccines. Dr Sandra Percell is an independent consultant working for Novartis Vaccines.

Words: 3,403 
112 Results: Immunological equivalence of the two lots of $4 \mathrm{CMenB}$ was established at

113 1-month after second vaccination. At baseline, $\leq 7 \%$ of participants had hSBA titres

$114 \geq 5$ to all the three reference strains. Two weeks following the second dose of

115 4CMenB, all participants had hSBA titres $\geq 5$ against fHbp and NadA compared with 84-96\% against the PorA antigens. At 1-month, corresponding proportions were $99 \%$, 118 similar adverse event profiles.

\begin{abstract}
:
Background: For decades, a broadly effective vaccine against serogroup B Neisseria meningitidis (MenB) has remained elusive. Recently, a four-component recombinant vaccine $(4 \mathrm{CMenB})$ has been developed and is now approved in Europe, Canada, Australia and some Latin American countries. This phase III, randomized study evaluated the lot consistency, early immune responses and the safety profile of 4CMenB in 11 to 17-year-old adolescents in Australia and Canada (NCT01423084).
\end{abstract}

Methods: In total, 344 adolescents received two doses of one of 2 lots of 4CMenB, 1month apart. Immunogenicity was assessed before, 2-weeks and 1-month following the second vaccination. Serum bactericidal activity using human complement (hSBA) was measured against three reference strains specific for the vaccine antigens Neisseria adhesin A (NadA), factor $\mathrm{H}$ binding protein (fHbp) and porin A (PorA) containing outer membrane vesicle (OMV). Responses to the Neisseria heparin binding antigen (NHBA) were assessed with an enzyme linked immunosorbent assay (ELISA). Local and systemic reactions were recorded for 7 days after each vaccination; unsolicited adverse events were monitored throughout the study. . 
120 Conclusions: Two doses of $4 \mathrm{CMenB}$ had an acceptable safety profile and induced a

121 robust immune response in adolescents with peak antibody responses observed at 14

122 days after vaccination. While a substantial non-uniform antigen-dependent early

123 decline in antibody titers was seen thereafter, a significant percentage of participants

124 continued to maintain protective hSBA titers at 1-month.

125

126 Funding: Novartis Vaccines

127

128 Word Count $=\mathbf{3 0 2}$ 


\section{Introduction}

130 Over the last two decades, substantial progress has been made in reducing the burden

131 of disease caused by Neisseria meningitidis through vaccination. Successful mono-

132 and multivalent vaccines against serogroups $\mathrm{A}, \mathrm{C}, \mathrm{W}$ and $\mathrm{Y}$ have been developed

133 based on serogroup-specific capsular polysaccharides and enhanced with

134 polysaccharide-protein conjugate technology. However, this vaccine strategy cannot

135 be employed for serogroup B, the last of the five major pathogenic meningococcal

136 groups, due to the capsule's structural homology to human fetal neural tissues,

137 resulting in poor immunogenicity [1, 2]. Serogroup B Neisseria meningitidis (MenB)

138 is now the leading cause of meningococcal disease in infants and young children,

139 accounting for over $80 \%$ of cases in Australia and some Latin American countries,

140 over 70\% in Europe, $66 \%$ in the UK, 50-80\% in Canada and one-third in the USA [3-

$1417]$.

143 Previously, tailor-made MenB vaccines have been developed from strain-specific

144 outer membrane vesicles (OMV) and successfully used to combat homologous strains

145 in clonal outbreaks [8-11]. However, due to limited or no effectiveness against

146 heterologous strains they are not suitable for use as a general serogroup B vaccine

147 [12]. Recently however, important advances have been made in the quest for a

148 universal MenB vaccine with the identification of conserved sub-capsular

149 meningococcal proteins [13, 14].

150

151 One four-component recombinant vaccine, $4 \mathrm{CMenB}$, contains three recombinant

152 proteins: factor $\mathrm{H}$ binding protein (fHbp), Neisserial adhesin $\mathrm{A}(\mathrm{NadA})$ protein and

153 Neisseria heparin binding antigen (NHBA), along with porin A (PorA) containing 
154 OMV derived from meningococcal NZ98/254 strain (previously used to control a

155 MenB clonal outbreak in New Zealand (NZ), the main PorA antigen is P1.4) [15].

156 Since development, the $4 \mathrm{CMenB}$ vaccine has been administered in phase I, and

157 pivotal phase IIb and III studies to over 8000 adults, adolescents and infants and has

158 been shown to be immunogenic, as measured by serum bactericidal assay using

159 human complement (hSBA), to a majority of reference strains within hypervirulent

160 clusters responsible for MenB disease [16-18].

161

162 This study measured baseline immunity, and the consistency and kinetics of immune

163 response following a two-dose schedule of two lots of 4CMenB (manufactured at

164 different sites) in healthy Australian and Canadian adolescents. Safety and tolerability

165 were also assessed.

166 
Methods

\section{Study design and participants}

169 The study (NCT01423084) was a phase III, multicentre, observer-blind, randomized

170 trial which involved healthy 11 to 17 -year-old adolescents across five centres in

171 Australia and seven in Canada between August and December 2011. Potential

172 participants were identified via advertising fliers in school newsletters or on

173 community noticeboards and from participant databases at the research centres.

174 Exclusion criteria were: previous receipt of MenB vaccine; previous meningococcal

175 disease (or household or intimate contact with an individual with N. meningitidis);

176 recent significant acute or chronic infection or fever $\geq 38.0^{\circ} \mathrm{C}$; recent antibiotic use;

177 known immunodeficiency or use of immunosuppressive doses of corticosteroids;

178 recent receipt of any blood products including immunoglobulin, planned receipt of

179 other vaccines within 30 days (within 14 and 60 days for influenza and live viral

180 vaccines respectively) or allergy to vaccine components. Participants were also

181 excluded if they were pregnant, breast feeding or unwilling to use acceptable birth

182 control measures within 30 days prior and 60 days following enrolment. A negative

183 pregnancy test was required for all females prior to receipt of each dose of $4 \mathrm{CMenB}$

184 vaccine. Written assent was obtained from each adolescent and written informed

185 consent was obtained from participant's parents or legal guardians. Approvals were

186 obtained from ethics committees at each participating research centre.

187

188 Both lots of the investigational vaccine 4CMenB had identical composition but were

189 formulated with OMV manufactured by Novartis Vaccines at two different sites in

190 Italy: Rosia (Lot 1) and Siena (Lot 2). 
192 Participants were randomized in a 1:1 ratio to receive two doses of either Lot 1 or Lot

1932 of 4 CMenB vaccine, one month apart. Blood samples (maximum $20 \mathrm{~mL}$ ) were

194 taken on day 1 before the first vaccination and 30 days after the second vaccination.

195 At pre-selected sites, an extra blood sample was taken two weeks following the

196 second dose in all participants to investigate the kinetics of the early immune response

197 after vaccination (Table 2). Participants were observed for at least 30 minutes after

198 each study vaccination. Local and systemic reactions and adverse events (AE) were

199 collected on a diary card for seven days following each vaccination. Serious adverse

200 events (SAE), medically attended AEs and AEs that resulted in a participant's

201 withdrawal from the study were collected throughout the study period.

202

203 Serological responses

204 Assays were performed at the Clinical Laboratory Science, Novartis Vaccines

205 Marburg, Germany. Sera were tested for bactericidal activity using a human

206 complement source (hSBA), against each of the three N. meningitidis serogroup B

207 reference strains: 44/76-SL (fHbp), 5/99 (NadA) and NZ98/254 (PorA) using the

208 method described previously ( ). These strains were chosen as each is mismatched for

209 all but one of the vaccine antigens, therefore each strain assesses the response to a

210 single vaccine component (44/76-SL - fHbp, 5/99- NadA and NZ98/254-PorA). The

211 IgG antibody concentration to the NHBA antigen were assessed using an enzyme-

212 linked immunosorbent assay (ELISA). At the time of the study, no suitable strain for

213 NHBA responses had been identified therefore an NHBA-specific ELISA was used

214 against vaccine antigen 287-953 ( ).

215

216 Statistical analysis 
217 The primary objective was to demonstrate equivalence of Lot 1 to Lot 2 of 4CMenB,

218 as measured by hSBA geometric mean titre (GMT) against 3 reference strains (44/76-

219 SL, 5/99 and NZ98/254) and ELISA geometric mean concentration (GMC) IgG

220 against the NHBA, 30 days after a primary course of 2 doses administered one month

221 apart. Equivalence was defined as the two-sided $95 \%$ confidence interval (CI) of the

222 ratio of the hSBA GMTs and GMCs being contained within the interval $(0.5,2.0)$.

223 The primary safety objective was to evaluate the safety and tolerability of two doses

224 of two lots of 4CMenB given one month apart in healthy adolescents.

226 The secondary immunogenicity objectives were to: assess the increase in hSBA GMT

227 and ELISA GMC (post- to pre-vaccination); and to calculate the percentage of

228 participants in each lot with $\mathrm{hSBA} \geq 1: 5$ one month following the second vaccination

229 for each of the three reference strains (44/76-SL, 5/99 and NZ98/254). Further, in a

230 subset of approximately 160 participants ( 80 per lot), the same outcome measures

231 were assessed two weeks following the second vaccination.

233 For each lot and each reference strain and for the NHBA antigen, the GMT (or GMC)

234 and two-sided 95\% CI was calculated. These were obtained from a two-way analysis

235 of variance (ANOVA) with factors for vaccine lot and study centre. For the

236 percentage of subjects with $\mathrm{hSBA} \geq 1: 5$, the associated 95\% Clopper-Pearson

237 confidence interval (CI) was measured. No between-group comparisons were

238 performed for any immune measure besides the primary endpoint, because the

239 analysis was underpowered to demonstrate equivalence. Titres and concentrations

240 below the limit of detection ( 2 for hSBA and 40 for ELISA) were set to half that limit

241 (i.e: 1 for hSBA and 20 for ELISA) for the purpose of analysis. 
243 The planned sample size of 135 evaluable participants (160 minus $15 \%$ attrition) per

244 arm, was calculated to provide $94 \%$ overall power to demonstrate consistency of the

245 immune response to the two lots of $4 \mathrm{CMenB}$ assuming a two-sided $\alpha=0.05$, an

246 underlying ratio of the vaccine group GMTs or GMCs of 1.0 for each of the reference

247 strains, and an equivalence interval of $(0.5,2.0)$. For an underlying ratio of the

248 vaccine group GMTs (or GMCs) of 1.1 for each strain, the overall power to

249 demonstrate consistency was calculated as approximately $90 \%$. 


\section{Results}

Of the 344 participants enrolled into the study, 170 were included in Lot 1 (Rosia) and 174 in Lot 2 (Siena) of whom 99\% and 98\% of participants completed the study, respectively. The per-protocol immunogenicity population (299 participants) included adolescents with no major protocol violations who provided sera for testing (147 and 152 participants from Lot 1 and 2, respectively) (Figure 1). The safety population comprised 342 participants (169 participants from Lot 1 and 173 from Lot 2). The demographic and baseline characteristics were well matched except for gender. The mean age at enrolment was 13.7 years $[ \pm 1.9]$ (Table 1$)$. The per protocol population for the extra blood taken two weeks following the second vaccination (visit 2) consisted of 76 and 71 participants in Lot 1 and 2, respectively.

At baseline, of 299 participants, $6(2 \%)$ had hSBA titres $\geq 5$ to the reference strains for fHbp (44/76-SL), 20 (7\%) for NadA (5/99) and 5 (2\%) for PorA (NZ98/254).

\section{Primary objective}

One month following the second vaccination, the ratios of hSBA GMTs in Lot 1 to Lot 2 were 1.0, 0.92 and 0.81 for each of the 3 reference strains 44/76-SL, 5/99 and NZ98/254 respectively, with corresponding CI of $(0.82,1.23),(0.77,1.1)$ and $(0.6$, 1.09) (Table 2). The Lot 1 to Lot 2 ratio of the ELISA GMCs against the NHBA, one month following the second vaccination, was 0.83 (95\% CI: 0.67, 1.02) (Table 2). Statistical equivalence of the two vaccine lots was met, as each ratio and CI were contained within the interval 0.5 and 2.0.

\section{Secondary objectives}


Immune response one month following the second vaccination:

289 The proportion of participants with $\mathrm{hSBA} \geq 1: 5$, one month following the second dose 290 of 4CMenB vaccine, were generally similar for reference strains 44/76-SL and 5/99

291 in both vaccine lots (99\% each and 100\% each, respectively). For the NZ98/254

292 strain, a higher proportion of participants in Lot 2 (79\%, CI 72-86) presented with

$293 \mathrm{hSBA} \geq 1: 5$ compared to Lot $1,70 \%$ (62-77) In addition (although statistically

294 equivalent), the ELISA GMR to baseline against NHBA antigen was slightly higher

295 in Lot 2 (153 (CI 131-179) compared to Lot 1 (122 (103-143). Reverse cumulative

296 distribution (RCD) functions of hSBA titres were similar for the two groups (Lot 1

297 and 2) for each of the 3 reference strains 44/76-SL, 5/99 and NZ98/254 and for

298 ELISA concentrations for NHBA antigen, both at baseline and at one month

299 following the second vaccination.

300

301 Immune response at two weeks following the second vaccination:

302 In the subset population, two weeks and one month following 4CMenB, $100 \%$ of

303 participants in both Lot 1 and Lot 2 groups attained hSBA $\geq 1: 5$ against reference

304 strains 44/76-SL and 5/99. However against strain NZ98/254, only 84\% and $96 \%$ of

305 adolescents achieved an hSBA titre $\geq 5$ at 2 weeks, and these proportions fell to $64 \%$

306 and $80 \%$ by 1 month following the second vaccination, for Lot 1 and Lot 2,

307 respectively (Figure 2).

308

309 In general, for both Lot 1 and Lot 2 vaccines, there was a substantial but non-uniform

310 decline in hSBA GMT levels for the 3 reference strains and ELISA GMCs against the

311 NHBA antigen, from two weeks to one month following the second vaccination

312 (Table 2). RCD curves of the hSBA titres and ELISA concentrations at two weeks 
313 and at one month after second vaccination are shown in Figure 1. For both lots, and

314 for hSBA titres against each reference strain and ELISA concentrations against

315 NHBA antigen, separation (shift to the left) is noted between the RCD at two weeks

316 and one month following the second vaccination, indicating a decline in immune

317 response between these 2 time-points.

318 No meaningful differences were observed in the pattern of the immune responses at 319 any time point, when analyzed by center and country

\section{Safety and Tolerability}

322 Overall, both lots of $4 \mathrm{CMenB}$ had similar safety profiles and were generally well 323 tolerated. Almost all adolescents (98\% in Lot 1 and 99\% in Lot 2) reported at least 324 one local, systemic or other reaction during the 7 days after either vaccination. The 325 proportion of participants with local reactions was similar between groups (96\% Lot 1 326 and $98 \%$ Lot 2). Most local and systemic reactions were mild to moderate in nature 327 (Tables 3 and 4). The most frequently reported local reaction after any vaccination 328 was pain (96\% and 98\%), reported as severe in $14 \%$ and $17 \%$ of Lot 1 and 2 329 participants, respectively. The most frequently reported solicited systemic reaction 330 was myalgia (59\% and $68 \%$ ), followed by headache ( $44 \%$ and $51 \%)$ and fatigue $(44 \%$ 331 and 49\%) in Lot 1 and 2 participants, respectively. Fever $\geq 38^{\circ} \mathrm{C}$ was infrequently 332 reported (5\% and 3\% in Lot 1 and 2 participants respectively). One participant in Lot 3332 withdrew from the study on day 34 due to an adverse event (infectious 334 mononucleosis) that started in day 14, with moderate severity and was judged as 335 unrelated to the study vaccine by the investigator. No death, serious adverse event or 336 pregnancy occurred. 
338

339

353 This is the first study to measure the early immune response following 4CMenB

354 vaccination. It was postulated that immune responses may remain static or decline

355 slightly from 2 to 4 weeks following 4 CMenB vaccination in adolescents. However, a

356 substantial decline over the two weeks was not expected. The finding of the peak

357 antibody response at 14 days following immunization is similar to that reported

358 following meningococcal serogroup $\mathrm{C}$ conjugate vaccine, Haemophilus influenzae

359 type b (Hib) polysaccharide and Hib conjugate vaccines (peak at 10-14 days), which

360 subsequently wane [19-21]. Recently, measurement of the longer term kinetics of

361 immune responses following the second dose of $4 \mathrm{CMenB}$ vaccine in university 
362 students has shown ongoing waning of hSBA titers from 1-month to 11-months

363 following vaccination [22].

365 Also of note, we found a difference in the rate of decline of hSBA titres between the

366 strains from two weeks to one month following the second $4 \mathrm{CMenB}$ vaccination. The

367 largest declines (a fall in hSBA GMT of 48\%) were observed for reference strains

368 5/99 and NZ98/254 against the NadA and PorA vaccine antigens respectively. The

369 PorA antigen also generated the lowest peak immune response of the four vaccine

370 components (as determined by fold-rise from baseline to two weeks following the

371 second vaccination and proportion of adolescents generating titres considered

372 protective, $\mathrm{hSBA} \geq 5$ ). Lower seroresponses to this same PorA antigen (NZ98/254)

373 have previously been reported. Following three doses of the monovalent MenB OMV

374 vaccine (MeNZB) used to help control a clonal outbreak in New Zealand: only 74\%

375 of 6-8 month-old [23], 75\% of 16-24 month-old [24] and 74-79\% of 8-12 year-old

376 children [25] developed a seroresponse, as assessed at 4 weeks after dose 3 (four-fold

377 rise in hSBA titre compared to baseline). Age-dependent antibody decline against this

378 PorA vaccine antigen was also described; 7 months following the third dose of

379 MeNZB in 6-8 month-old infants only $28 \%$ had persistent $\mathrm{hSBA} \geq 4$; compared to

$38036 \%, 14$ months following the third dose in 8-12 year-old children; and 50\%, 10

381 months following the third dose in adults [26]. Accordingly, as the rate of decline of

382 hSBA titres (or waning of antibody) is differential according to age and strain, it is

383 possible that susceptibility to meningococcal infection and duration of vaccine

384 protection might be differential according to age and geographical region (depending

385 on the expression of proteins in the circulating disease-causing strains). Importantly,

386 vaccine efficacy has been shown to mirror a rapid decline in circulating bactericidal 
antibodies following vaccination with monovalent MenB OMV vaccines in Norway and New Zealand [27, 28] and with MenC conjugate vaccines in the UK [29].

This study highlights the complexity in assessing immunogenicity and determining generalizability of the four-component MenB vaccine for different geographical regions. At baseline, pre-existing immunity to the meningococcal vaccine antigens in our Australian and Canadian adolescent population was low (2\%-7\%) of adolescents had hSBA titres $\geq 5$ to strains for fHbp, NadA and PorA antigens, respectively, compared to $34 \%-44 \%$ with hSBA titres $\geq 4$ in a prior $4 \mathrm{CMenB}$ adolescent study conducted in Santiago and Valparaiso, Chile [17]. In the current study, while 99-

$397100 \%$ of adolescents achieved protective titres $(\mathrm{hSBA} \geq 5)$ to reference strains $44 / 76-$ 398 SL and 5/99, one month following the second 4CMenB vaccination, only $75 \%$ did so 399 for the NZ98/254 strain. In comparison, in the Chilean study, 99-100\% of adolescents 400 achieved hSBA titres $\geq 4$ (albeit a slightly less conservative measure) to each of these 4013 reference strains [17]. The difference in baseline immunogenicity between the two 402 similar 11-17 year-old adolescent vaccine-naïve populations could partially relate to 403 assays conducted in to different laboratories, but may more likely be due to different

404 levels of acquisition of natural immunity though nasopharyngeal carriage in each 405 population. Higher baseline immunogenicity levels may suggest higher levels of 406 environmental exposure to the meningococcal vaccine strains in Chilean than in the 407 Canadian and Australian populations. Indeed, even accounting for different 408 laboratories, the RCD function curves of hSBA responses for the reference strain 409 NZ98/254 (PorA) in Chilean adolescents, who received the accelerated two-dose 410 schedule (one month apart), were noticeably shifted to the right (higher hSBA titres) 411 compared to the current study. However hSBA titres were similar against reference 
412 strains 44/76-SL and 5/99 in both studies [17]. Further, Chilean adolescents with

413 higher baseline levels (hSBA titres $\geq 4$ ) generated higher post vaccination antibody

414 titres than vaccine antigen naïve adolescents (hSBA titres $<4$ at baseline) and these

415 differences persisted up to 6 months [17]. Recently, assays done in a subset of

416 University students in a UK meningococcal carriage study found high baseline

417 immunity to the $4 \mathrm{CMenB}$ vaccine antigens, similar to the Chilean study (these assays

418 were also performed at Public Health England laboratory, UK) [22] together with

419 high rates of carriage (33\%) [30]. In comparison, meningococcal carriage rates are

420 low in Canada. No carriage data are available for Australia. Accordingly, in

421 populations with low baseline immunity to MenB, persistence of antibody may be

422 reduced. The possibility of an additional vaccine impact on carriage may further

423 amplify these differences in population-specific effects.

425 The hSBA GMTs and ELISA GMCs at one month after second vaccination in the two

426 vaccine groups were statistically equivalent for each of the three MenB reference

427 strains for vaccine antigens fHbp, NadA PorA and NHBA antigen, respectively. This

428 finding was not unexpected. Both vaccine lots were identical in composition, but

429 formulated with OMV manufactured at two different facilities.

431 There was a slightly higher frequency of systemic reactions reported by adolescents

432 who received Lot 2 vaccine (compared to Lot 1). Overall, the rate of reported

433 systemic reactions was slightly higher in our study than previously reported, although

434 rates of fever $\geq 38^{\circ} \mathrm{C}$ were similar [17]. Of note, in both studies the vast majority of

435 reported systemic reactions were of mild or moderate severity, a similar number were 
reported as severe and no evidence of increasing rates of reactions with subsequent dosing was identified [17].

The $4 \mathrm{CMenB}\left(\right.$ Bexsero $^{\circledR}$ ) vaccine was licensed for use in the European Union in January 2013, and subsequently in Australia, Canada and some Latin American countries, for individuals from 2 months-of age [31]. In March 2014, the UK became the first country to recommend its use in a National Immunization Program (at 2, 4 and 12 months of age) [32] where coverage of $4 \mathrm{CMenB}$ is estimated at $88 \%$ of invasive MenB strains [33] (subject to vaccine pricing and cost-effectiveness requirements by the UK Government). In addition, a second MenB vaccine, a bivalent vaccine containing recombinant factor $\mathrm{H}$ binding protein variants (also called rLP2086), manufactured by Pfizer, has undergone Phase II clinical trials [34-36] and was licensed in the US in October 2014 for use in adolescents and young adults. Both 4CMenB and rLP2086 had received Breakthrough Therapy designation from the US FDA. In January 2015, 4CMenB was also approved in United States (US) as a 2-dose series in adolescents 11- 25 years ( ) .

In conclusion, this study demonstrated the lot to lot consistency of $4 \mathrm{CMenB}$ vaccine manufactured at different sites. Two doses of $4 \mathrm{CMenB}$, given one month apart, had an acceptable safety profile and induced robust immune responses in adolescents, though waning of titers was observed from 2 weeks to 1 month after vaccination. Further, this study's finding of differential baseline antibody titres to $4 \mathrm{CMenB}$ vaccine antigens compared with age-matched but geographically distinct populations, affecting immunogenicity, is important. Population-based post-implementation 
460 disease surveillance to assess the potential differential vaccine impact (on herd 461 immunity and duration of protection) in each region will be imperative.

462 


\section{Acknowledgements}

464

465 We are grateful to the participants of this study and their family members. We thank

466 the contributions of the staff members of the Vaccine and Immunisation Research

467 Group (Melbourne), Marita Kefford, Sharon Trevorrow, Mairead Phelan, Annmarie

468 McEvoy, Jane Ryrie, Clare Brophy, Janet Briggs, Marie West, Jacinta Sonego, Jacinta

469 O'Keefe, Judith Spotswood, Paula Nathan and Bernie McCudden; Dr Tanya Stoney,

470 Caroline Talbot and Jennifer Kent of the Vaccine Trials Group (Perth); Chris Heath,

471 Susan Lee, Sue Evans, Trinh Tran, Mary Walker, of the Vaccinology and

472 Immunology Research Trials Unit and Dr. Raymond Chuk, Dr. Uyen Doan, Mr.

473 Aaron Buckner of the Queensland Paediatric Infectious Diseases Clinical Trials

474 Centre (Canada). Graphical support was provided by Dr Shivani Vadapalli (Novartis

475 Vaccines).

476

477

478

479 


\section{References}

[1] Wyle FA, Artenstein MS, Brandt BL, Tramont EC, Kasper DL, Altieri PL, et al. Immunologic response of man to group B meningococcal polysaccharide vaccines. The Journal of infectious diseases. 1972;126:514-21.

[2] Finne J, Leinonen M, Makela PH. Antigenic similarities between brain components and bacteria causing meningitis. Implications for vaccine development and pathogenesis. Lancet. 1983;2:355-7.

[3] Lahra MM, Enriquez RP. Annual report of the Australian Meningococcal Surveillance Programme, 2011. Communicable diseases intelligence. 2012;36:E25162.

[4] Halperin SA, Bettinger JA, Greenwood B, Harrison LH, Jelfs J, Ladhani SN, et al. The changing and dynamic epidemiology of meningococcal disease. Vaccine. 2012;30 Suppl 2:B26-36.

[5] Safadi MA, Cintra OA. Epidemiology of meningococcal disease in Latin America: current situation and opportunities for prevention. Neurological research. 2010;32:263-71.

[6] Bettinger JA, Scheifele DW, Le Saux N, Halperin SA, Vaudry W, Tsang R. The disease burden of invasive meningococcal serogroup B disease in Canada. The Pediatric infectious disease journal. 2013;32:e20-5.

[7] Report HP. Invasive meningococcal disease (laboratory reports in England): 2013/2014 annual data by epidemiological year. 23 January 2015.

[8] Sierra GV, Campa HC, Varcacel NM, Garcia IL, Izquierdo PL, Sotolongo PF, et al. Vaccine against group B Neisseria meningitidis: protection trial and mass vaccination results in Cuba. NIPH Ann. 1991;14:195-207; discussion 8-10. [9] Bjune G, Hoiby EA, Gronnesby JK, Arnesen O, Fredriksen JH, Halstensen A, et al. Effect of outer membrane vesicle vaccine against group $B$ meningococcal disease in Norway. Lancet. 1991;338:1093-6.

[10] Holst J, Feiring B, Naess LM, Norheim G, Kristiansen P, Hoiby EA, et al. The concept of "tailor-made", protein-based, outer membrane vesicle vaccines against meningococcal disease. Vaccine. 2005;23:2202-5.

[11] Oster P, Lennon D, O'Hallahan J, Mulholland K, Reid S, Martin D. MeNZB: a safe and highly immunogenic tailor-made vaccine against the New Zealand Neisseria meningitidis serogroup B disease epidemic strain. Vaccine. 2005;23:2191-6.

[12] O'Hallahan J, McNicholas A, Galloway Y, O'Leary E, Roseveare C. Delivering a safe and effective strain-specific vaccine to control an epidemic of group B meningococcal disease. N Z Med J. 2009;122:48-59.

[13] Pizza M, Scarlato V, Masignani V, Giuliani MM, Arico B, Comanducci M, et al. Identification of vaccine candidates against serogroup B meningococcus by wholegenome sequencing. Science. 2000;287:1816-20.

[14] Fletcher LD, Bernfield L, Barniak V, Farley JE, Howell A, Knauf M, et al. Vaccine potential of the Neisseria meningitidis 2086 lipoprotein. Infection and immunity. 2004;72:2088-100.

[15] Arnold R. Poisson Regression Modelling of the Effectiveness of the Meningococcal B Vaccine (MeNZB) Updated results to December 2008, Technical Report. New Zealand Ministry of Health2010. p. 90.

[16] Gossger N, Snape MD, Yu LM, Finn A, Bona G, Esposito S, et al. Immunogenicity and tolerability of recombinant serogroup B meningococcal vaccine administered with or without routine infant vaccinations according to different immunization schedules: a randomized controlled trial. Jama. 2012;307:573-82. 
[17] Santolaya ME, O'Ryan ML, Valenzuela MT, Prado V, Vergara R, Munoz A, et al. Immunogenicity and tolerability of a multicomponent meningococcal serogroup $B$ (4CMenB) vaccine in healthy adolescents in Chile: a phase $2 \mathrm{~b} / 3$ randomised, observer-blind, placebo-controlled study. Lancet. 2012;379:617-24. [18] Vesikari T, Esposito S, Prymula R, Ypma E, Kohl I, Toneatto D, et al. Immunogenicity and safety of an investigational multicomponent, recombinant, meningococcal serogroup B vaccine (4CMenB) administered concomitantly with routine infant and child vaccinations: results of two randomised trials. Lancet. 2013;381:825-35.

[19] Borrow R, Southern J, Andrews N, Peake N, Rahim R, Acuna M, et al. Comparison of antibody kinetics following meningococcal serogroup $\mathrm{C}$ conjugate vaccine between healthy adults previously vaccinated with meningococcal A/C polysaccharide vaccine and vaccine-naive controls. Vaccine. 2001;19:3043-50. [20] Madore DV, Johnson-Kraines CL, Rothstein EP, Smith DH. Kinetics of antibody response to Haemophilus influenzae type b vaccines. Pennridge Pediatric Associates. Curr Med Res Opin. 1999;15:105-12.

[21] de Voer RM, van der Klis FR, Engels CW, Schepp RM, van de Kassteele J, Sanders EA, et al. Kinetics of antibody responses after primary immunization with meningococcal serogroup $C$ conjugate vaccine or secondary immunization with either conjugate or polysaccharide vaccine in adults. Vaccine. 2009;27:6974-82.

[22] Dull PB, X. Bazaz R et al. Serum bactericidal antibody levels following quadrivalent conjugate

(MenACWY-CRM) or serogroup B (4CMenB) meningococcal vaccines in a Phase 3 study

to evaluate the effect of vaccination on pharyngeal carriage of N. meningitidis in young adults. Meningitis and Septicaemia in Children and Adults 2013. London, England, UK2013.

[23] Jackson C, Lennon DR, Sotutu VT, Yan J, Stewart JM, Reid S, et al. Phase II meningococcal B vesicle vaccine trial in New Zealand infants. Archives of disease in childhood. 2009;94:745-51.

[24] Wong S, Lennon D, Jackson C, Stewart J, Reid S, Crengle S, et al. New zealand epidemic strain meningococcal B outer membrane vesicle vaccine in children aged 16-24 months. The Pediatric infectious disease journal. 2007;26:345-50. [25] Hosking J, Rasanathan K, Mow FC, Jackson C, Martin D, O'Hallahan J, et al. Immunogenicity, reactogenicity, and safety of a P1.7b,4 strain-specific serogroup B meningococcal vaccine given to preteens. Clin Vaccine Immunol. 2007;14:1393-9. [26] Jackson C, Lennon D, Wong S, Yan J, Stewart J, Reid S, et al. Antibody persistence following MeNZB vaccination of adults and children and response to a fourth dose in toddlers. Archives of disease in childhood. 2011;96:744-51. [27] Holst J, Feiring B, Fuglesang JE, Hoiby EA, Nokleby H, Aaberge IS, et al. Serum bactericidal activity correlates with the vaccine efficacy of outer membrane vesicle vaccines against Neisseria meningitidis serogroup B disease. Vaccine. 2003;21:734-7.

[28] Galloway Y, Stehr-Green P, McNicholas A, O'Hallahan J. Use of an observational cohort study to estimate the effectiveness of the New Zealand group B meningococcal vaccine in children aged under 5 years. Int J Epidemiol. 2009;38:4138.

[29] Trotter CL, Andrews NJ, Kaczmarski EB, Miller E, Ramsay ME. Effectiveness of meningococcal serogroup $C$ conjugate vaccine 4 years after introduction. Lancet. 2004;364:365-7. 
579 [30] Read Rea. Impact of a quadrivalent conjugate (MenACWY-CRM) or a

580 serogroup B (4CMENB) meningococcal vaccine on meningococcal carriage in

581 English university students. ESPID 21032013.

582 [31] Novartis. Novartis receives EU approval for Bexsero®, first vaccine to prevent

583 the leading cause of life-threatening meningitis across Europe. 2013.

584 [32] JCVI Position statement on use of Bexsero meningococcal B vaccine in the UK.

5852014.

586 [33] Frosi G, Biolchi A, Lo Sapio M, Rigat F, Gilchrist S, Lucidarme J, et al.

587 Bactericidal antibody against a representative epidemiological meningococcal

588 serogroup B panel confirms that MATS underestimates 4CMenB vaccine strain

589 coverage. Vaccine. 2013;31:4968-74.

590 [34] Richmond PC, Marshall HS, Nissen MD, Jiang Q, Jansen KU, Garces-Sanchez

591 M, et al. Safety, immunogenicity, and tolerability of meningococcal serogroup B

592 bivalent recombinant lipoprotein 2086 vaccine in healthy adolescents: a randomised,

593 single-blind, placebo-controlled, phase 2 trial. Lancet Infectious Diseases.

594 2012;12:597-607.

595 [35] Marshall HS, Richmond PC, Nissen MD, Wouters A, Baber J, Jiang Q, et al. A

596 phase 2 open-label safety and immunogenicity study of a meningococcal B bivalent

597 rLP2086 vaccine in healthy adults. Vaccine. 2013;31:1569-75.

598 [36] Vesikari T D-DJ, Ostergaard L, et al. Safety, Tolerability, and Immunogenicity

599 of an Investigational Meningococcal Serogroup B Bivalent rLP2086 Vaccine When

600 Administered in Regimens of 2 or 3 Doses in Healthy Adolescent Subjects aged 11 to

60118 Years. Poster presented at: 9th Conference of the Meningitis Research Foundation;

6022013 November 5-6. London, United Kingdom2013.

603 [37] Donnelly J, Medini D, Boccadifuoco G, Biolchi A, Ward J, Frasch C, et al.

604 Qualitative and quantitative assessment of meningococcal antigens to evaluate the

605 potential strain coverage of protein-based vaccines. Proceedings of the National

606 Academy of Sciences of the United States of America. 2010;107:19490-5.

607 [38] Vogel U, Taha MK, Vazquez JA, Findlow J, Claus H, Stefanelli P, et al.

608 Predicted strain coverage of a meningococcal multicomponent vaccine (4CMenB) in

609 Europe: a qualitative and quantitative assessment. The Lancet Infectious diseases.

$610 \quad 2013 ; 13: 416-25$.

611 [39] Predicted coverage of MenB strains indicate the potential of BEXSERO to

612 impact MenB disease. 2014.

613 